\title{
COMPACT WAVEGUIDE DISTRIBUTION WITH ASYMMETRIC SHUNT TEES FOR THE EUROPEAN XFEL
}

\author{
V. Katalev, S. Choroba, DESY, Hamburg, Germany
}

\begin{abstract}
In the European X-ray FEL 32 superconducting cavities ( 4 cryomodules with 8 cavities per module) are connected to one $10 \mathrm{MW}$ multibeam klystron through a waveguide distribution system. The XFEL tunnel has limited space for the waveguide system and therefore a new more compact waveguide distribution has been developed. The waveguide distribution is based on a binary cell which consists of two circulators connected to a shunt tee with integrated phase shifters. Four binary cells are combined by three asymmetric pretunable shunt tees. The asymmetric shunt tees allow to change the RF power for each pair of cavities and to reach the maximum cryomodule gradient. In this paper we will present the status of the waveguide distribution system and report on the development of the different new waveguide components.
\end{abstract}

\section{INTRODUCTION}

The linac for the XFEL has 27 RF stations. Each RF station consists besides of other components of a $10 \mathrm{MW}$ klystron and a power distribution for 4 cryomodules with 8 cavities each [1]. The linac is operated at an RF pulse length of $1.38 \mathrm{~ms}$ with $0.77 \mathrm{~ms}$ filling time, a repetition rate of $10 \mathrm{~Hz}$ and an RF frequency of $1.3 \mathrm{GHz}$. The superconducting cavity has a loaded quality factor of about $4.6 \times 10^{6}$.

In order to accelerate $5 \mathrm{~mA}$ beam with gradient 23.6 $\mathrm{MV} / \mathrm{m}$ it is necessary to supply $122 \mathrm{~kW}$ RF power to each cavity. Not more then 5.2 MW RF power from the klystron is required including losses in the waveguide distribution system and a regulation reserve of $15 \%$ for phase and amplitude control. When operated at $10 \mathrm{MW}$ the klystron supplies $280 \mathrm{~kW}$ to each cavity. All waveguide components of the RF distribution will be designed for such power level.

\section{WAVEGUDE DISTRIBUTION LAYOUT}

The XFEL project is based on one tunnel conception [1]. Therefore to save tunnel space and also to increase reliability and decrease mounting time of the waveguide distribution in the XFEL tunnel some new waveguide components have been developed.
The new $350 \mathrm{~kW}$ circulators with integrated dummy load (so named isolators) have been planned for use in the distribution. The isolators have to be cooled by demineralised water.

The phase shifters are placed between the klystron and the isolators and therefore the maximal voltage for phase shifters is reduced by factor two. By combining the two phase shifters with classic shunt tee in one device we decrease the amount of flanges.

The distance between two cavities in the cryomodule is $1383.6 \mathrm{~mm}$. To phase the waveguide distribution system for the beam so called fixed phase shifters have been designed. A fixed phase shifter is a rectangular waveguide which has another cross section then the standard WR650. This allows to compensate a phase delay in the standard waveguide.

The asymmetric shunt tees can be pretuned with coupling ratio in the $1-9 \mathrm{~dB}$ range at the DESY side. This makes it possible to run each cryomodule with maximal average gradient and thereby avoid a possible problem in case of a 'weak' cavity.

The bellows are needed to compensate the production tolerances, thermal expansion and to decrease mechanical stress for the power coupler of superconducting cavity

The new compact waveguide distribution for XFEL cryomodule (see Fig.1) is based on binary cells. The binary cell consists of two isolators and symmetric shunt tee with two integrated phase shifters. The binary cells are connected together by asymmetric shunt tees and fixed phase shifters. Therefore the waveguide distribution system for the XFEL cryomodule consists of only 8 isolators and bellows, 4 shunt tees with integrated phase shifters, 3 asymmetric pretunable shunt tees, 3 fixed phase shifters and one $\mathrm{H}$ bend. All of the waveguide components are placed in one plane. The number of waveguide elements and flanges has been reduced by 1000 or 2000 respectively compared with the first proposal of a standard FLASH/TTF like distribution for the XFEL [2]. The waveguide distribution has compact dimensions and a small weight. Therefore the waveguide distribution can be preassembled with a specific cryomodule and might even be transported together with it to the XFEL tunnel.

All of waveguide components are made from

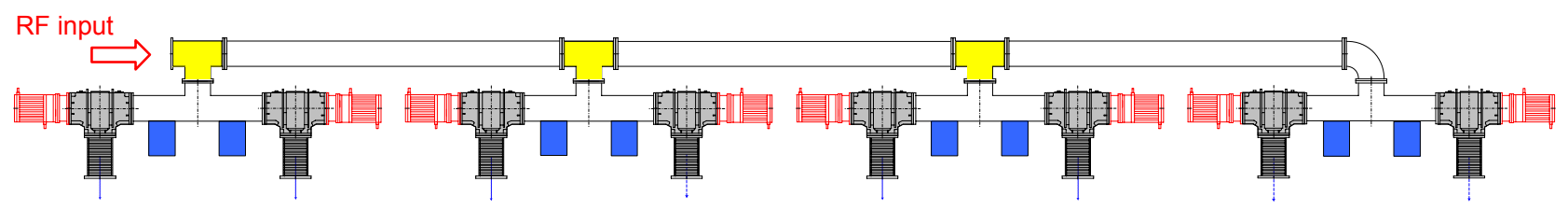

Figure 1: RF distribution with asymmetric shunt (yellow) tees and shunt tees with integrated phase shifters (blue) for a cryomodule with 8 cavities. 
aluminium alloy with flanges PDR14 or UDR14.

\section{WAVEGUDE DISTRIBUTION COMPONENTS}

\section{Binary Cell}

A basic element of the power waveguide distribution is the binary cell (see Fig.2) which consists of a pair of isolators connected together by a long shunt tee. The distance between outputs is $1383.6 \mathrm{~mm}$ or zero degree phase shift for the beam. Because the binary cell has a symmetric plane the phase shift of 0 degree is automatically achieved.

\section{Shunt Tee with Integrated Phase Shifters}

A distance between the flanges of two isolators in the binary cell is $940 \mathrm{~mm}$ which is enough to place two movable pistons of $370 \mathrm{~mm}$ length. The piston has no sliding contacts and is isolated from the inner surface of the waveguide by kapton foil or polyethylene of $0.15 \mathrm{~mm}$ thickness. By moving in the piston up to $25 \mathrm{~mm}$ the phase is changed by 90 degree for each output arm of the shunt tee.

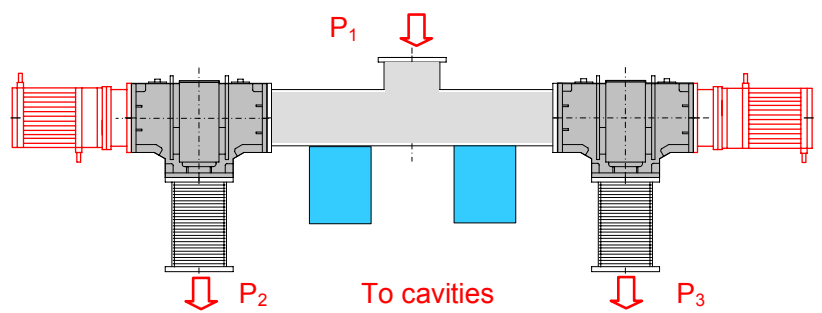

Figure 2: The binary cell consists of a pair circulators with dummy load connected together by shunt tee with integrated phase shifters.

By placing the phase shifters before the isolators we decrease the maximal voltage two times and thereby the reliability of the distribution is increased. But in this case the SWR of the binary cell is more sensitive to a change of piston position in the phase shifters.

The shunt tee with integrated phase shifters has first been simulated with MicroWave Studio (MWS). Then it has been tested with success up to $800 \mathrm{~kW}$ input power. The input SWR did not exceed 1.2.
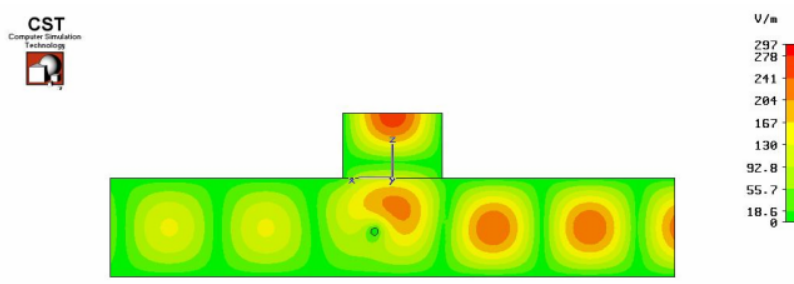

Figure 3: Output RF power of the shunt tee with the post shifted relatively the symmetry plane.
The shunt tee also can be tuned for different output power $\mathrm{P}_{2}$ and $\mathrm{P}_{3}$ by moving of the post inside of the shunt tee (see Fig.3). Results of MWS simulation are shown Fig.4 and Fig.5.

For reasonable $\mathrm{SWR}<1.2$ the output RF power for two cavities can be tuned in the range of $6 \mathrm{~dB}$ and therefore it

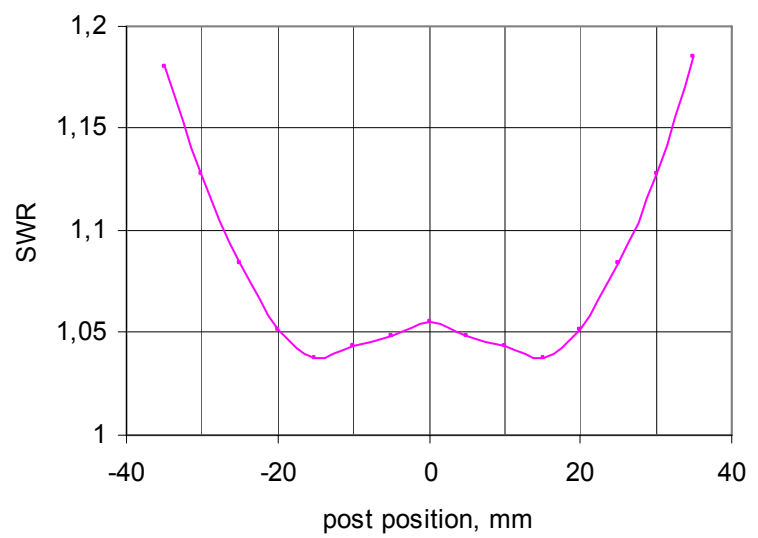

Figure 4: Input SWR of shunt tee with different position of the post relative to the symmetry plane.

would be possible to operate the cavities with a different gradient a factor of about two.

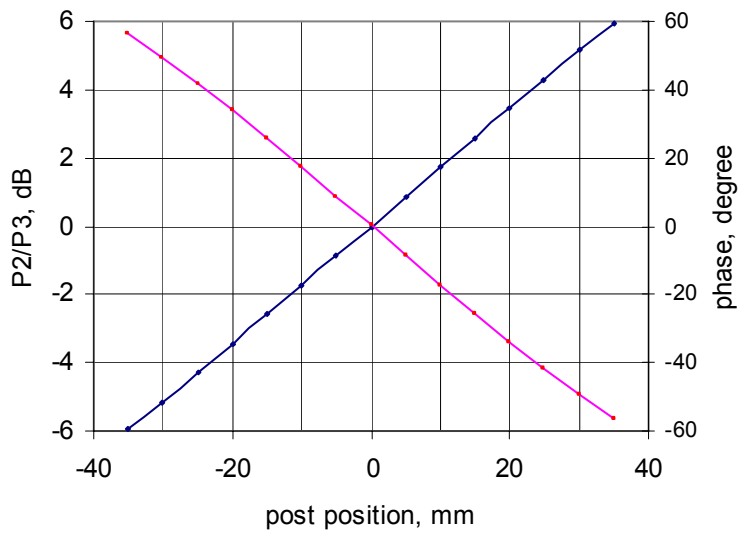

Figure 5: Relation for output power $\mathrm{P}_{2}$ to $\mathrm{P}_{3}$ (blue line) and phase shift (red line) between the arms.

At the same time the difference of the output phases of the tee and thus at the cavities changes by up to 55 degrees (see Fig.5). The integrated phase shifters compensate this phase difference. They also compensate other phase shifts due to mechanical, thermal and RF deviations.

\section{Pretunable Asymmetric Shunt Tee}

To supply pair cavities with required RF power an asymmetric shunt tee has been developed (see Fig.6). The asymmetric shunt tee is an H-plane T-junction with two inductive posts inside. By moving the posts the input power splits into two outputs with the required ratio (see Fig.7) at a reasonable input matching (Fig.8). 


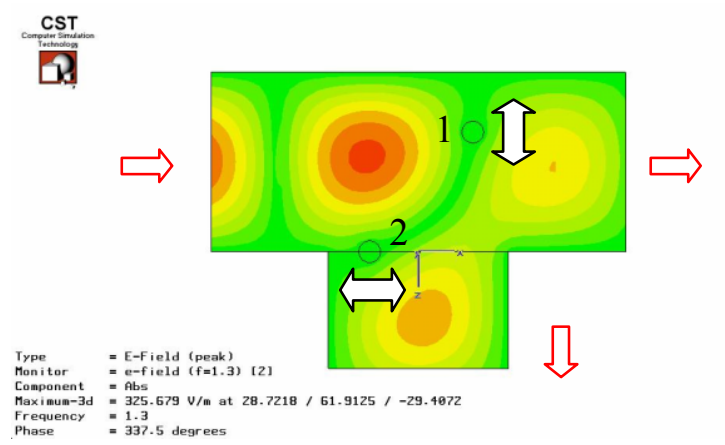

Figure 6: Pretunable asymmetric shunt tee with two inductive posts

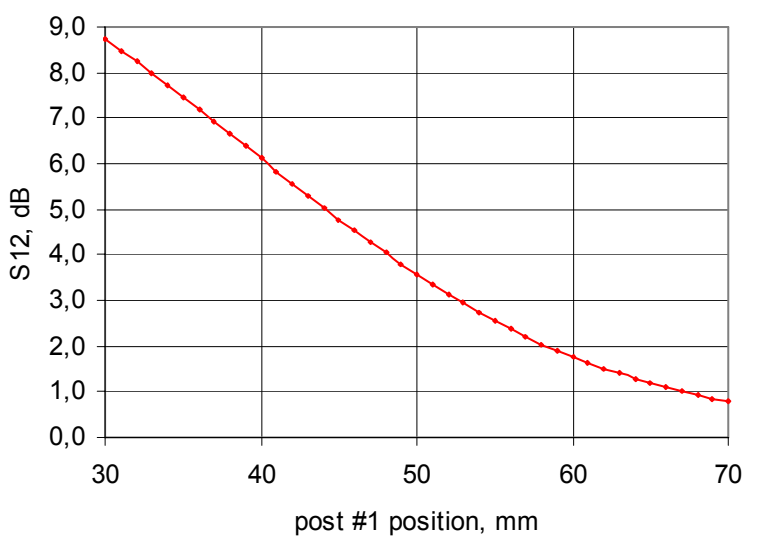

Figure 7: Coupling ratio of the asymmetric shunt tee as function position of post \#1

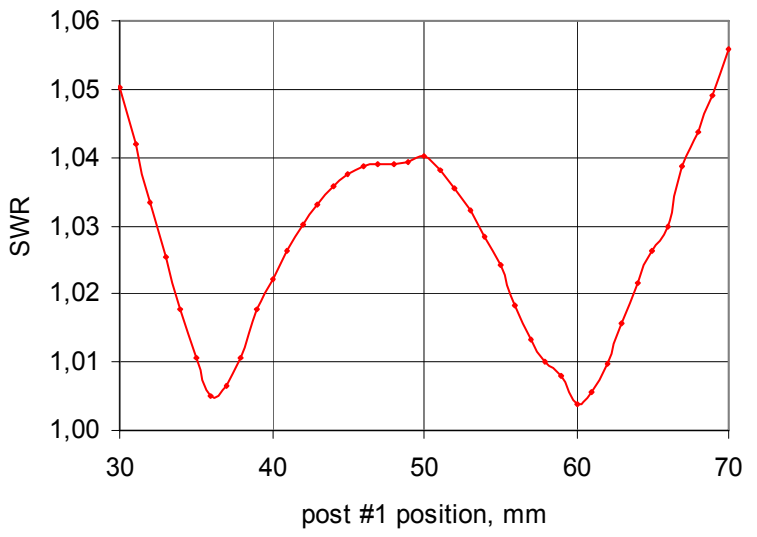

Figure 8: Input SWR of the asymmetric shunt tee as function position of post \#1

The installation of two posts is simple and fast and therefore the asymmetric shunt tee can be tuned exactly for each pair of cavities in advance. By tuning beforehand the RF power for each binary cell the maximal average gradient for an entire cryomodule can be increased. This reduces a possible problem with "weak" cavity.

\section{Fixed Phase Shifter}

The asymmetric shunt tees are connected by $2387 \mathrm{~mm}$ long straight waveguides. In this case the phase shift between the binary cells is about - 220 degrees. In order to compensate this phase shift the waveguides with non standard cross section are used. The waveguide with width $153.1 \mathrm{~mm}$ has a phase delay of 220 degree compare with standard WR650. To reduce input SWR of such fixed phase shifter from 1.15 down to 1.04 the waveguide height is decreased from $82 \mathrm{~mm}$ to $78 \mathrm{~mm}$. For the waveguide with reduced cross section the specific losses increases from $0.0075 \mathrm{~dB} / \mathrm{m}$ to $0.009 \mathrm{~dB} / \mathrm{m}$.

By using the fixed phase shifters the waveguide distribution can be phased with tolerance of \pm 10 degrees. And this tolerance can be compensated by the integrated tunable phase shifters of the binary cell.

\section{SUMMARY}

- A new compact waveguide distribution has been developed at DESY for the XFEL. The first prototype will be installed in the middle of 2007 for cryomodule \#6 at the FLASH linac.

- The new waveguide distribution is a pretunable system. It allows to avoid the "weak" cavity problem and each cryomodule can run with maximal average gradient.

- Integrated waveguide components are used for the distribution system to decrease the quantity of flanges and to increase the reliability of the whole RF system. All new waveguide components have been tested successfully at the high power RF stand on the DESY side.

- The waveguide distribution is a 2D system and therefore takes minimal place in the XFEL tunnel. It can be preassembled beforehand for a specific cryomodule before installation in the tunnel.

\section{ACKNOWLEDGMENTS}

We would like to express our gratitude to F.Eints, J.Rahnhahn, I.Sandvoss and other of our colleges of the MHF-p group for testing of a lot of waveguide components.

\section{REFERENCES}

[1] R. Brinkmann, "Accelerator Layout of the XFEL", LINAC04, Lübeck, Germany, August 16-20, 2004

[2] V. Katalev, S. Choroba, "Waveguide distribution systems for European XFEL", EPAC06, Edinburgh, Scotland, June 26-30, 2006 